\title{
An Open Formula for the Numerical Integration of First Order Differential Equations
}

1. Introduction. The integration scheme given below combines the accuracy of multi-point formulas with the convenience, lack of starter formulas, etc., of two-point formulas, as described, for example, by Milne [1]. The price paid is the necessity of evaluating the right hand side of

$$
y^{\prime}=f(x, y)
$$

at points which may lie outside the range of integration. It is the writer's belief that this is compensated for by the ease of programming the scheme for a digital computer.

2. The Integration Formulas. For each $n=3,4,5, \cdots$, there exists a matrix $D_{n}$ of order $n$ and rank $n-1$ such that if $\bar{y}=\left(y_{0}, y_{1}, \cdots, y_{n-1}\right)$ is a vector of values of the function $y(x)$ at equally spaced points $x_{0}, x_{0}+h, \cdots, x_{0}+(n-1) h$, then $\bar{y}^{\prime}=\left(y_{0}{ }^{\prime}, y_{1}{ }^{\prime}, \cdots, y_{n-1}^{\prime}\right)$ is given by

$$
\bar{y}^{\prime}=D_{n} \bar{y}+\bar{E}
$$

where $\bar{E}$ is an error vector whose elements are $O\left(h^{n-1} y^{(n)}(\xi)\right)$.

Recurrence formulas for the propagation of the solution of (1) can be obtained by setting

$$
D_{n} \bar{y}+\bar{E}=f(\bar{x}, \bar{y})
$$

where

$$
f(\bar{x}, \bar{y}) \equiv\left(f\left(x_{0}, y_{0}\right), f\left(x_{1}, y_{1}\right), \cdots, f\left(x_{n-1}, y_{n-1}\right)\right),
$$

from which all but two variables can be eliminated successively, yielding the desired recurrence relation. Thus for $n=3$,

$$
\begin{aligned}
& y_{0}^{\prime}=\frac{1}{2 h}\left(-3 y_{0}+4 y_{1}-y_{2}\right)+\frac{h^{2}}{3} y^{(3)}=f\left(x_{0}, y_{0}\right) \\
& y_{1}^{\prime}=\frac{1}{2 h}\left(-y_{0}+y_{2}\right)-\frac{h^{2}}{6} y^{(3)}=f\left(x_{1}, y_{1}\right) \\
& y_{2}^{\prime}=\frac{1}{2 h}\left(y_{0}-4 y_{1}+3 y_{2}\right)+\frac{h^{2}}{3} y^{(3)}=f\left(x_{2}, y_{2}\right) .
\end{aligned}
$$

Solving (4.2) for $y_{2}$, inserting in (4.1) we get

$$
y_{1}-y_{0}=\frac{h}{2}\left\{f\left(x_{0}, y_{0}\right)+f\left(x_{1}, y_{1}\right)\right\}-\frac{h^{3}}{12} y^{(3)}(\xi),
$$

the familiar trapezoidal rule. 
With $n=4$ we eliminate $y_{2}, y_{3}$ from

$$
\begin{aligned}
& y_{0}^{\prime}=\frac{1}{6 h}\left(-11 y_{0}+18 y_{1}-9 y_{2}+2 y_{3}\right)-\frac{h^{3}}{4} y^{(\mathrm{IV})}=f\left(x_{0}, y_{0}\right) \\
& y_{1}^{\prime}=\frac{1}{6 h}\left(-2 y_{0}-3 y_{1}+6 y_{2}-y_{3}\right)+\frac{h^{3}}{12} y^{(\mathrm{IV})}=f\left(x_{1}, y_{1}\right) \\
& y_{2}^{\prime}=\frac{1}{6 h}\left(y_{0}-6 y_{1}+3 y_{2}+2 y_{3}\right)-\frac{h^{3}}{12} y^{(\mathrm{IV})}=f\left(x_{2}, y_{2}\right) \\
& y_{3}^{\prime}=\frac{1}{6 h}\left(-2 y_{0}+9 y_{1}-18 y_{2}+11 y_{3}\right)+\frac{h^{3}}{4} y^{(\mathrm{IV})}=f\left(x_{3}, y_{3}\right)
\end{aligned}
$$

getting

$$
\begin{aligned}
y_{1}-y_{0} & =\frac{h}{12}\left[5 f\left(x_{0}, y_{0}\right)+8 f\left(x_{1}, y_{1}\right)-f\left(x_{2}, y_{2}\right)\right]+\frac{h^{4}}{24} y^{(\mathrm{IV})} \\
y_{2} & =5 y_{0}-4 y_{1}+2 h\left\{f\left(x_{0}, y_{0}\right)+2 f\left(x_{1}, y_{1}\right)\right\}+\frac{h^{4}}{6} y^{(\mathrm{IV})} .
\end{aligned}
$$

Replacing $y_{2}$ in (7.1) by (7.2) and using the mean value theorem we get finally

$$
\begin{aligned}
& y_{1}-y_{0}=\frac{h}{12}\left\{5 f\left(x_{0}, y_{0}\right)+8 f\left(x_{1}, y_{1}\right)-f\left(x_{2}, y_{2}^{*}\right)\right\} \\
& O \frac{h^{4}}{24} y^{(\mathrm{IV})}(\xi)\left[1+\frac{h}{3} \frac{\partial f}{\partial y}\left(x_{2}, \eta\right)\right]
\end{aligned}
$$

where

$$
\begin{gathered}
y_{2} *=5 y_{0}-4 y_{1}+2 h\left[f\left(x_{0}, y_{0}\right)+2 f\left(x_{1}, y_{1}\right)\right] \\
x_{0} \leq \xi \leq x_{2}
\end{gathered}
$$

and $\eta$ lies between $y_{2}$ and $y_{2}{ }^{*}$.

If $f(x, y)$ is linear in $y$ then $y$ is given explicitly by (8.1) and (8.2); otherwise an initial guess may be made of $y_{1}, y_{2}{ }^{*}$ computed from (8.2), and a new $y_{1}$, computed from (8.1), repeating the process until convergence is reached before proceeding to the next point.

In the linear case, (1) takes the form

$$
y^{\prime}(x)=P(x)+Q(x) y(x),
$$

and (8.1), (8.2) become

$$
\begin{aligned}
y_{1} & {\left[1-\frac{h}{3}\left(2 Q_{1}+Q_{2}\right)+\frac{h^{2}}{3} Q_{1} Q_{2}\right]-y_{0}\left[1+\frac{h}{12}\left(Q_{0}-Q_{2}\right)-\frac{h^{2}}{6} Q_{0} Q_{2}\right] } \\
& =\frac{h}{12}\left[5 P_{0}+8 P_{1}-P_{2}\right]-\frac{h^{2} Q_{2}}{6}\left[P_{0}+2 P_{1}\right]+\frac{h^{4}}{24} y^{(\mathrm{IV})}(\xi)\left[1+\frac{h}{3} Q_{2}\right] .
\end{aligned}
$$


The method clearly generalizes to $n>4$ yielding formulas of higher order accuracy. The involved calculations necessary in carrying out the propagation, however, may make the method too inconvenient for machine calculation.

3. An example. We illustrate the method with the solution of

$$
\left\{\begin{aligned}
y^{\prime} & =1+y \\
y(0) & =2
\end{aligned}\right.
$$

for which the exact solution is $y=3 e^{x}-1$.

The functions $P(x), Q(x)$ are here identically unity, and (10) becomes

$$
\left[1-h+\frac{h^{2}}{3}\right] y_{1}=\left[1-\frac{h^{2}}{6}\right] y_{0}+h-\frac{h^{2}}{2}
$$

or

$$
y_{1}=A\left(1+y_{0}\right)-1
$$

where

$$
A=\left[1-\frac{1}{6} h^{2}\right]\left[1-h+\frac{1}{3} h^{2}\right]^{-1} \text {. }
$$

\begin{tabular}{|c|c|c|c|}
\hline$x$ & $y$ (Runge-Kutta) & $y$ (Eq. 10) & $y$ (Exact) \\
\hline $\begin{array}{l}0.00 \\
0.20 \\
0.40 \\
0.60 \\
0.80 \\
1.00\end{array}$ & $\begin{array}{ll}2.00000 & 0000 \\
2.66420 & 4603 \\
3.47546 & 5123 \\
4.46633 & 9967 \\
5.67659 & 6021 \\
7.15480 & 4623\end{array}$ & $\begin{array}{ll}2.00000 & 0000 \\
2.66420 & 4351 \\
3.47546 & 4508 \\
4.46633 & 8842 \\
5.67659 & 4190 \\
7.15480 & 1828\end{array}$ & 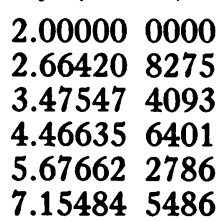 \\
\hline
\end{tabular}

The fourth order Runge-Kutta method also gives (13) with

$$
A=\left[1+h+\frac{1}{2} h^{2}+\frac{1}{6} h^{3}\right] \text {. }
$$

For $h=.05$ the solution was propagated twenty steps with the following results:

4. Significance. In order to obtain accuracy of higher order than the third, using the Runge-Kutta technique, it is necessary to evaluate the functions $Q(x)$, $P(x)$ in equation (9) at points interior to each mesh interval. On the other hand, equation (10) requires that evaluation only at points at which $y$ is to be calculated, plus one extra beyond the right hand limit of integration.

This fact, plus the freedom from starter formulas required by some multi-point methods combine to enhance the value of equation (10), or equivalently (8.1), (8.2) for machine computation.

HERBERT S. WILF

Nuclear Development Corporation of America White Plains, New York

1. W. E. MILNE, Numerical Calculus. Approximations, Interpolation, Finite Differences, Numerical Integration, and Curve Fitting, Princeton Univ. Press, New Jersey, 1949, p. 96-98. 